\title{
Conceptual survey of phase modulation based wavelength registered networks
}

\author{
M.-S. Kao
}

T.-C. Lin

Indexing terms: Multiwavelength network, Communications network

\begin{abstract}
The implementation of a dense wavelength-division multiplexing (WDM) based wide-area network inevitably involves a large number of semiconductor lasers requiring particular temperature and driving current control. The concept of a novel multiwavelength network that uses only one laser source to generate all the carriers needed for the entire network is investigated. The central idea is to use a stable reference laser to generate two sets of optical carriers as the 'carrier supplies' for all network nodes: one set for signal transmission and the other for signal reception. A promising feature of the proposed network is the wavelength registration, that is, all carrier wavelengths are unambiguously identified. This special feature is of importance for a standard wide-area multiwavelength network. Optical phase modulation is extensively employed in the carrier generation as well as in information delivery. A 40 -wavelength network is considered with each wavelength carrying 20 phase-modulated microwave subcarriers, a total throughput of $160 \mathrm{Gbit} / \mathrm{s}$ per single-mode fibre is estimated.
\end{abstract}

\section{Introduction}

Optical fibres are the obvious choice of medium for information transmission in future high-speed networks. The extremely low loss and virtually unlimited bandwidth of single-mode fibre enables applications both in long haul transmissions and local interconnections. In the future, the continuous progresses on $O / E$ components, highspeed modulation, optical amplification, photonic switching, as well as high-speed optical signal processing, will certainly change the scene of communication networks.

The steady progress on WDM technology makes channel spacing of the order of several gigahertz possible $[1,2]$. (Note here that WDM is not differentiated from optical FDM as they belong essentially to the same category.) With WDM technology, the bottle-neck imposed by the limited electronic bandwidth is removed by assigning the available bandwidth of a single-mode fibre to many wavelengths. Many experimental and theoretical

(C) IEE, 1994

Paper 9852J (E13), first received 30th March and in revised form 23rd August 1993

The authors are with the Department of Communication Engineering and Centre for Telecommunications Research, National Chiao Tung University, Hsinchu, 30050 Taiwan, Republic of China

IEE Proc.-Optoelectron., Vol. 141, No. I, February 1994 studies have indicated that very wide bandwidth optical networks can be accomplished by WDM technology [3-8]. On the other hand, the microwave subcarrier multiplexing (SCM) technique allows simultaneous transmission of many signals in digital or analogue form, thus offering a flexible transmission technology compared with traditional TDM systems [9, 10]. A promising feature inherent in a SCM system with optical phase modulation is the allowance of several geographically different nodes sharing an optical carrier via phase modulators [11]. This feature is useful in network applications. Another feature of the phase-modulated SCM system is the preservation of a constant envelope thereby easing optical amplification, particularly in multichannel systems.

WDM-based optical networks are expected to become widespread. In WDM networks, wavelength can be used not only for transmission but also for switching, routing, addressing or other network functions $[12,7,8]$. To construct a wide-area WDM network, stable light sources are required. Most of the proposed networks feature distributed-light-source architecture; i.e. each transmitting node has its own source for information delivery and thus there are a large number of light sources in the network. As semiconductor lasers are subject to driving current and temperature variation, it is very difficult to implement a stable wide-area multiwavelength network with a large number of widely distributed light sources.

In this paper, a conceptual study is made of a novel multiwavelength network that uses a single laser source to generate all the carriers needed for the entire network. The network is called the wavelength registered network (WRN) because carrier wavelengths are well identified without ambiguity all around the network. Instead of adopting the widespread distributed-light-source architecture, the WRN employs a centralised-light-source architecture to achieve wavelength registration. That is, all carriers in the WRN come from a common centre, called the wavelength generation centre (WGC), but are not locally generated at network nodes. In the WGC, a stable reference laser is used as the origin to generate two sets of optical carriers as the 'carrier supplies' for all network nodes. Because all carriers originate from a common reference laser, their wavelengths are well identified without ambiguity. Also, owing to the linearity of fibre-optic networks, carrier wavelengths remain unchanged as they travel around the network. Thus the WRN is wavelength-registered because all carrier wavelengths are well identified within the network. On the other hand, the combinating of WDM and SCM has been shown to be a promising approach for highcapacity fibre networking [7]. Phase modulation based SCM is therefore used here for information delivery. 


\section{2}

\section{Critical issues}

The basis of WRN is to use a centralised-light-source architecture to construct a wavelength-registered multiwavelength network. The architecture of the WRN for constructing a wide-area network is shown in Fig. 1.

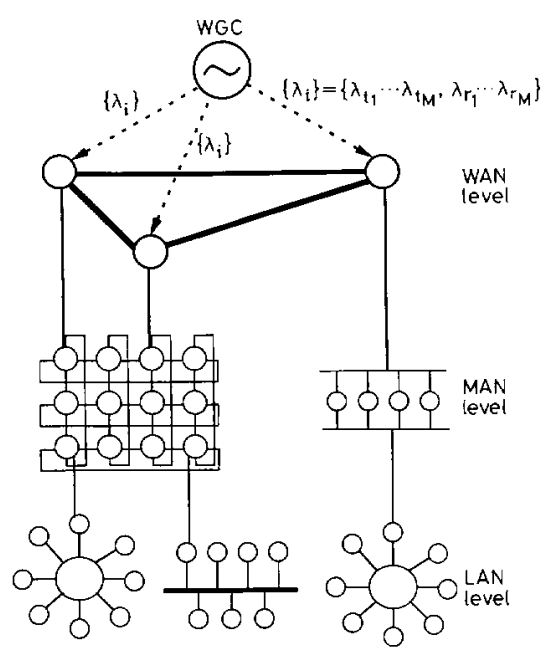

Fig. 1 Architecture of the WRN

The WGC generates two sets of carriers being used for the entire network. The WGC could be located at the centre of a wide-area network (WAN). The carriers are sent to all network nodes in the WAN, MANs, and LANs through a feeder network

Here the WGC generates carriers for the entire network and there is no carrier source in any network node. The carriers generated in the WGC are sent to nodes in the wide-area network (WAN), metropolitan-area network (MAN) and local-area network (LAN) through a feeder network. Each node then uses these carriers for information exchange through various communication networks. To implement such a network, several issues should be considered.

(a) The WGC is the centre where the carriers are generated. In the WRN, two sets of optical carriers, the transmission carrier set and the receiving carrier set, are generated. The two carrier sets each consist of $M$ equallyspaced optical carriers, which constitute the bases of the multiwavelength network. The transmission carrier set $\left\{\lambda_{t_{1}}, \ldots, \lambda_{t_{M}}\right\}$ is used for information transmission and the receiving carrier set $\left\{\lambda_{r_{1}}, \ldots, \lambda_{r_{M}}\right\}$ is used for information detection. As the two carrier sets constitute the network, they should be very stable, have no drift and have extremely narrow linewidths.

(b) The feeder network is the network distributing carriers from the WGC to all network nodes. This network is absent for a distributed-light-source network but is necessary for the WRN. The function of this network is simple: it distributes necessary carriers from the WGC to every network node. The distribution is hierarchical: the two carrier sets are first sent to distribution centres through single-mode fibres and are then further distributed to network nodes. Depending on the requirements, a node may receive one or more carriers out of $\left\{\lambda_{t}\right\}$ for transmitting information; similarly, it may receive carriers out of $\left\{\lambda_{r i}\right\}$ for information detection. In the feeder network, erbium-doped fibre amplifiers (EDFA) will probably be extensively used for compensating branch and path losses in distribution fibres [13-15]. The gain- bandwidth and the amplified spontaneous emission noise of EDFA are major problems. Possible nonlinear conversions occurring in the distribution fibres will also be considered.

(c) The communication network is the network performing information exchange among network nodes. At a transmitting node, information is put on to a transmitting carrier through optical phase modulation. Specifically, we adopt SCM that allows many nodes to share an optical carrier by sequential phase modulation [11]. This enables full utilisation of the carrier bandwidth and realises a flexible transmission. At the receiving end, we use a specific carrier in the receiving set $\left\{\lambda_{r i}\right\}$ to detect the phase-modulated SCM signal. The receiving carrier has effectively the same role as a local oscillator in a conventional coherent $S C M$ receiver, but it is provided by the WGC and is not locally generated. A polarisation diversity scheme can be used to overcome the problem caused by polarisation fluctuation in the transmitting and receiving carriers [16]. EDFA may be used in the communication network for signal amplification and possibly for receiving carrier amplification to ensure high receiver sensitivity [17].

(d) The photonic switching fabric provides a promising solution to resolving the bottleneck caused by limited electronic switching bandwidth. In a multiwavelength network, photonic switching through wavelength exchange is a promising switching fabric [12]. In such a switch, wavelength stability is critical. Fortunately, carrier wavelengths are very stable and are well identified in the WRN. Thus, a reliable photonic switching system can be implemented. This is not the case for a network without wavelength registration, in which wavelength stability and ambiguity will be rather cumbersome issues.

\subsection{The WGC}

The WGC is central to the WRN. Two sets of carriers are generated which will be distributed to network nodes for signal transmission and detection. The carriers generated should have stable and narrow linewidth outputs so that the impairment caused by wavelength drift and laser phase noise in phase-modulated systems can be minimised.

2.1.1 Frequency spacing: First frequency spacing between the carriers is characterised. A multiwavelength phase-modulated SCM transmitter is shown in Fig. 2 with the transmitting carriers $\left(f_{t_{1}}, \ldots, f_{t_{M}}\right)$ each carrying $N$ subcarriers $\left(f_{1}, \ldots, f_{N}\right)$. For illustrative purpose, in this

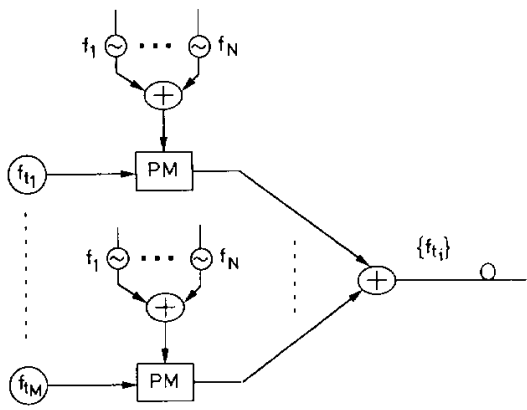

Fig. 2 Schematic diagram of a multiwavelength coherent SCM transmitter

$\mathrm{PM}=$ phase modulator

$f_{1}, \ldots, f_{N}=$ subcarrier frequencies

$f_{t 1}, \ldots, f_{t N}=$ optical carrier frequencies

IEE Proc-Optoelectron., Vol. 141, No. I, February 1994 
Section we use frequencies $\left\{f_{t}\right\}$ and $\left\{f_{r}\right\}$ instead of wavelengths $\left\{\lambda_{t_{i}}\right\}$ and $\left\{\lambda_{r_{i}}\right\} . N$ baseband signals are first modulated on the microwave subcarriers $\left(f_{1}, \ldots, f_{N}\right)$, then the combined SCM signal is modulated on an optical carrier via a phase modulator. Finally the phase modulator outputs are summed and are distributed to receiving nodes through single-mode fibres. The spectrum of the multiwavelength SCM signal is shown in Fig. 3. It follows from conventional phase-modulation theory that the subcarriers locate at both sides of the optical carrier with $180^{\circ}$ phase shift. Here the high-order terms are absent because they are negligible for low modulation index [10].

The information carried by a specific transmitting carrier is detected by a corresponding receiving carrier in $\left\{f_{r}\right\}$. Thus, there are equal number of carriers in both sets. The allocation of the receiving carrier is shown in Fig. 4. The arrangement uses $f_{r_{i}}$ to heterodyne-detect the information carried by subcarriers $\left(f_{t_{i}}+f_{1}, \ldots, f_{t_{i}}+f_{N}\right)$.

Let $B_{P D}$ denote the photodetector (PD) bandwidth. To ensure that cross-products between $f_{r_{i}}$ and $\left(f_{t_{i}}+f_{1}, \ldots\right.$, $f_{t_{i}}+f_{N}$ ) appear in the photocurrent, it is necessary to have

$$
B_{P D}>f_{r i}-\left(f_{i i}+f_{1}\right)
$$

On the other hand, to minimise cross-wavelength interference, we must have

$$
\left(f_{t_{i+1}}-f_{N}\right)-f_{r i}>B_{P D}
$$

so that the PD does not respond to the interference resulting from cross-products between $f_{\mathrm{r}_{\mathrm{i}}}$ and the subcarriers in $f_{u+1}$. Let $f_{I F}=f_{v_{i}}-f_{u}$ be the difference between the transmitting and receiving carriers. From the above two equations, the frequency spacing between two neighbouring transmitting carriers, denoted by $\Delta f_{t}$, is readily obtained as

$$
\Delta f_{t}>2 f_{I F}+f_{N}-f_{1}
$$

As an example, let the subcarrier frequencies range from 2.1 to $5.9 \mathrm{GHz}$ with $200 \mathrm{MHz}$ spacing [18] and we have an $8 \mathrm{GHz}$ PD bandwidth. If we take $f_{I F}=8 \mathrm{GHz}$ then the minimum value of $\Delta f_{t}$ is about $20 \mathrm{GHz}$. It is also clear from Fig. 4 that the receiving carriers have the same frequency spacing as the transmitting carriers.

In the WRN, we consider a system with $\Delta f_{t}=24 \mathrm{GHz}$ corresponding to a wavelength spacing of $0.2 \mathrm{~nm}$ around
$1.55 \mu \mathrm{m}$, and take the transmitting carrier set as $\lambda_{t_{1}}=$ $1552.2 \mathrm{~nm}, \lambda_{\mathrm{s}}=1552.4 \mathrm{~nm}, \ldots, \lambda_{\text {. }}=1560 \mathrm{~nm}$, a total of 40 carriers. The receiving set has the same number of carriers as the transmitting carrier with each shifted from the corresponding transmitting carrier by $f_{I F}=8 \mathrm{GHz}$. The subcarriers again range from 2.1 to $5.9 \mathrm{GHz}$ with $200 \mathrm{MHz}$ channel spacing, each carrying $200 \mathrm{Mbits} / \mathrm{s}$ digital data, and a total of $4 \mathrm{Gbit} / \mathrm{s}$ information. Thus in each fibre $160 \mathrm{Gbit} / \mathrm{s}$ information (40 wavelengths with each carrying $4 \mathrm{Gbit} / \mathrm{s}$ information) may be transmitted. This high capacity transmission is performed over a spectral range of only $8 \mathrm{~nm}$ in which EDFA have small signal gain over $20 \mathrm{~dB}$; many tunable optical devices can easily accommodate such a narrow spectral region.

2.1.2 The two-stage multicarrier generator: Once the carrier spacing is specified, the desired carriers must be generated. The crucial requirements in generating the carriers are stable output wavelengths and very narrow linewidths. These can be achieved by adopting a stable reference wavelength, possibly generated by a nonsemiconductor laser originated from atomic or molecular transition, for providing a stable and narrow linewidth wavelength reference. A straightforward way to generate the carrier set is to use the multisource configuration experimentally demonstrated in References 6 and 19. As multiple semiconductor sources are employed, a wide linewidth is expected and circumstantial control of many sources is necessary. A promising alternative by optical phase modulation was proposed in Reference 20 . By feeding an optical carrier into a phase modulator driven by a microwave oscillator, the resulting signal can be expressed as

$$
s(t)=A \sum_{n=-\infty}^{\infty} J_{n}(\beta) \cos \left[2 \pi\left(f_{c}+n f_{m}\right) t\right]
$$

where $f_{c}$ is the optical carrier frequency, $f_{m}$ is the microwave frequency, $\beta$ is the modulation index, and $J_{n}(\beta)$ are Bessel functions of the first kind. In this way many new optical carriers shifted from $f_{c}$ by integer multipliers of $f_{m}$ are generated. To generate the considered 40 carriers, a single phase modulator may be insufficient because of the weak high-order terms. However, multistage carrier generator can be applied to generate the desired carriers. For example, a two-stage multicarrier generator as shown in Fig. 5 , can be used. Let $f_{m}=24 \mathrm{GHz}$ be the desired fre-

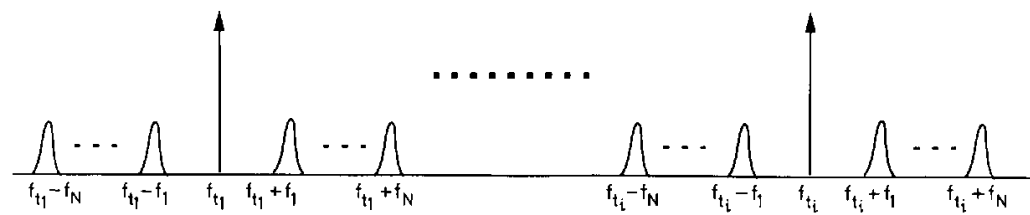

Fig. 3 Optical spectrum of the multiwavelength phase-modulated SCM signal

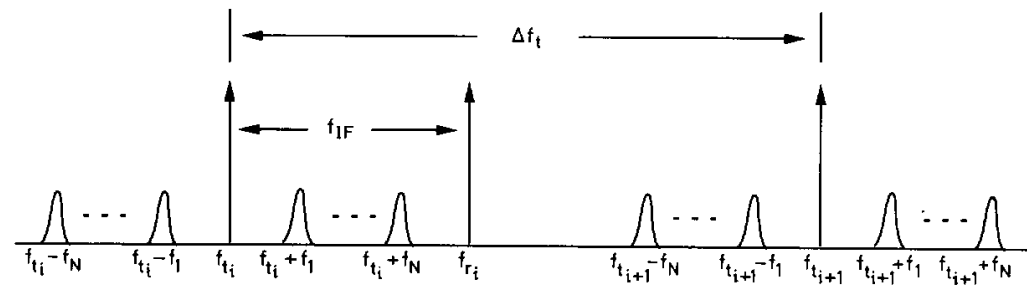

Fig. 4 Spectral allocation of the transmitting and the receiving carriers Frequency spacing $\Delta f_{t}=f_{t+1}-f_{t i}$ and $f_{I F}=f_{t i}-f_{t i}$ 
quency spacing. An optical carrier from the reference laser is first applied to a high-speed phase modulator driven by a microwave frequency $q f_{m}$, where $q$ is an integer and $q>1$. Thus at the output of the phase modulator, several newly generated carriers shifting from $f_{c}$ by integer multipliers of $q f_{m}$ are generated. For example, the input power is mainly disseminated over the $(2 k+1)$ carriers spreading from $f_{c}-k q f_{m}$ to $f_{c}+k q f_{m}$ with spacing $q f_{m}$. Here the number $k$ is determined by the modulation index. These carriers are filtered by optical filters, and are further fed to $(2 k+1)$ phase modulators driven by a microwave frequency $f_{m}$, each then producing $(2 j+1)$ carriers. It is easy to see that if we appropriately choose the modulation indexes to obtain the relationship $q=2 j+1$, we indeed have a total of $(2 k+1) q$ carriers spreading from $f_{c}-(k q+j) f_{m}$ to $f_{c}+(k q+j) f_{m}$ with spacing $f_{m}$. Let $q=5$ and $\beta=2$ for the first-stage phase modulator, then we have nine carriers (four at each side of the original carrier, $k=4$ ). In the second stage, we let $\beta=0.5$, then each phase modulator will generate five carriers $(j=2)$ and the relationship of $q=2 j+1$ is satisfied. Thus, we have a total of $(2 k+1) q=45$ carriers which is enough for our 40-carrier system.

\subsection{The feeder network}

The function of feeder network is to distribute the two carrier sets to network nodes. A wide-area WRN, such as a nationwide network, may consist of many subnetworks with a large number of nodes. To deliver the carriers from the WGC to network nodes, a reasonable approach is to first send the carriers to some distribution centres in the subnetworks and then distribute them to appropriate nodes. Each subnetwork may have one or more distribution centres, and the carriers needed in the subnetwork are provided by the centres.

There are two critical issues related to the feeder network. First, in distributing the carriers from the WGC to distribution centres or from distribution centres to network nodes, EDFAs are expected to be extensively used for compensating the path loss. Thus, the limited gain-bandwidth product of EDFA and the amplified spontaneous emission (ASE) noise should be considered. Fortunately, theoretical and experimental studies show that EDFA can accommodate a wide spectral region with more than $20 \mathrm{~dB}$ unsaturated optical gain [15], which is quite sufficient to amplify the considered 40 wavelength signals simultaneously within $8 \mathrm{~nm}$ spectral range. The results also show that there is almost no power penalty in the multichannel amplification compared with single channel amplification [15]. On the other hand, the ASE noise is expected to set a limit on the achievable signal-to-noise ratio at the receiving end if cascaded EDFAs are employed. However, because of the low-noise nature of EDFA, theoretical calculations indicate that the penalty incurred by accumulated ASE noises is small and will not seriously limit the capacity of a WDM system $[15,21]$. Thus, EDFA with good properties both in multichannel amplification and noise performance is ideal for carrier amplification in the feeder network of WRN.

Another issue of concern is the power loss and crosstalk caused by nonlinear optical effects in the distribution fibres [22]. Although a fibre-optic network is essentially a linear network, nonlinear conversions do exist in fibres and become significant when a strong optical power is launched into the fibre. Optical nonlinearities such as stimulated Brillouin scattering (SBS), stimulated Raman scattering (SRS), four-wave mixing (FWM) and carrier induced phase modulation (CIP) are likely to be present. SBS is caused by the interaction between optical photons and acoustic phonons; this induces new optical waves. A critical power at which system performance degrades lies on the order of several milliwatts [23]. Because in a multichannel system each channel interacts independently with the fibre, the critical power level is independent of channel number. SRS arises from the interaction between incident photons and lattice vibrations. Because of the wide Raman bandwidth, Raman interaction is unavoidable in multichannel systems and results in power transfer from the shorter wavelength channels to the longer wavelength channels [24]. Using the calculated results of Reference 23 , the allowable transmitted power for a 40-channel system for $1 \mathrm{~dB}$ power penalty is larger than that of SBS. FWM results from the mixing of two or more waves to produce new carriers, leading to power loss of the transmitted carrier as well as interference in a multichannel system. FWM is a rather complicated effect which depends on the number of channels and on the fibre dispersion parameter. From the example given in Reference 23, the critical power value for FWM is about $1 \mathrm{~mW}$ for a 40-channel system. CIP is a result of the intensity-dependent refractive index in optical fibres. This causes self-phase modulation in a single-channel system and cross-phase modulation in a multichannel

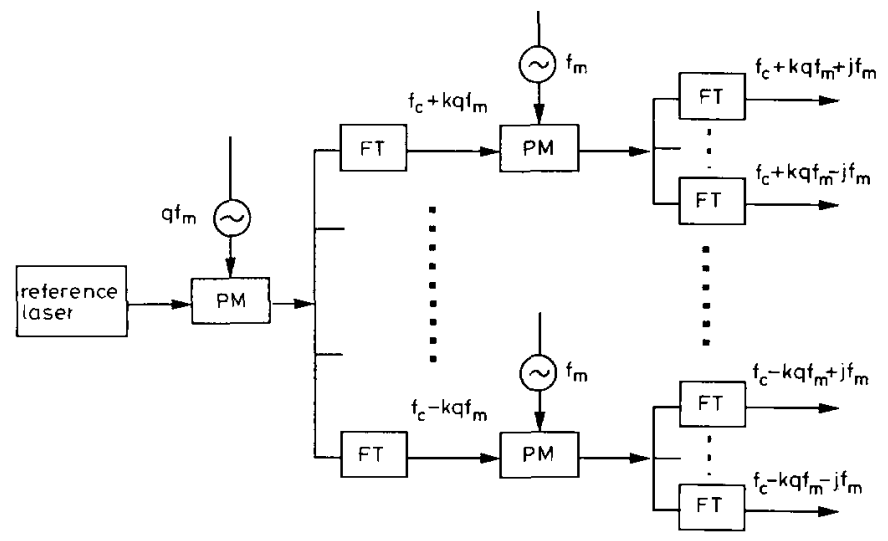

Fig. 5 A two-stage multicarrier generator using optical phase modulation $F T=$ fixed-frequency filter $q$ is an integer and $q>1$ 
system. Theoretical study showed that the phase fluctuation owing to cross-phase modulation is proportional to the square root of channel number and is usually much larger than that of self-phase modulation [25]. If the power fluctuation of the transmitted carrier is small, it was indicated that the limitation owing to CIP will be negligible even for a larger number of channels [23]. As the carriers distributing in the feeder network are essentially in $\mathrm{CW}$ form with very little intensity fluctuation, the penalty caused by CIP will be negligible.

In summary, the limitation imposed by optical nonlinearities is to limit the transmitted power of the carriers in the feeder network to less than about $1 \mathrm{~mW}$. This can be achieved easily, first by limiting the launched carrier powers in the feeder network to be less than the critical value and secondly by limiting the gain of EDFA in compensating the path loss not to over-compensate the carrier power. Once the carrier power is limited to below the critical value, there is little nonlinear conversion during the transmission path of the feeder network.

\subsection{Communication networks}

The communication network functions in a different way from the feeder network, using the available carriers for information delivery. Here we study several possible communication network structures.

Fig. 6 shows a single wavelength ring (logical) network which provides transparent interconnection between $N$ nodes at geographically different locations. Here we use just one of the carriers to form the small network. Information is put on to a specific transmitting carrier $\lambda_{t_{a}}$ by sequentially modulating a series of phase modulators with each being assigned a unique subcarrier frequency [11]. The corresponding receiving wavelength $\lambda_{r}$ is fed to each receiver as a means of converting the phase information into photocurrent, and RF tuning is performed subsequently to select one of the subcarrier's information. Therefore, each receiver is able to access all the other nodes thus forming a transparent network. Here advantage has been taken of SCM modulation for sharing an optical carrier by $N$ nodes.
The above network can be expanded to a large transparent network as shown in Fig. 7. In this case, each transmitting wavelength from the distribution centre is used to accommodate $N$ nodes as before, using sequential phase modulation. Then, the modulated carriers $\left\{\lambda_{t ;}^{\prime}\right\}$ are combined by a star coupler and fed back to all receivers.

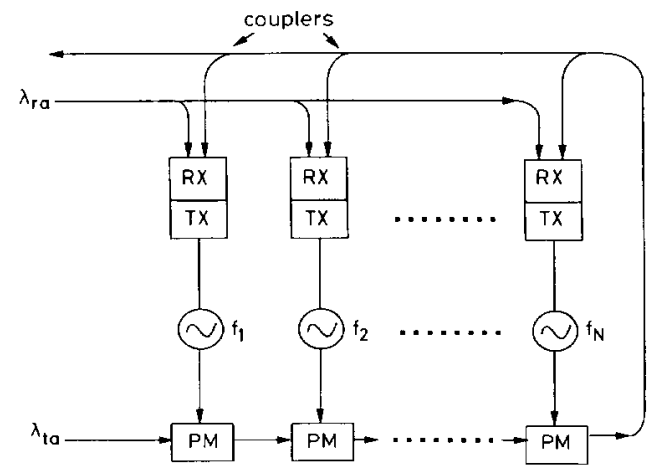

Fig. 6 Schematic diagram of a single wavelength transparent network $\lambda_{10}=$ transmitting carrier $\lambda_{r a}=$ receiving carrier
$\mathbf{P M}=$ phase modulator

Each receiver accepts the receiving carrier set $\left\{\lambda_{r_{i}}\right\}$ coming from the distribution centre with a tunable optical filter (not shown in the Fig. 7) placed at the front end. The filter selects one of the receiving carrier to detect the information carried by a specific transmitting wavelength. A recently demonstrated wavelength-tunable liquid crystal filter can serve the purpose [26]. The filter has a wide tuning range $(1.47-1.6 \mu \mathrm{m})$, capable of selecting one of 600 channels with $0.2 \mathrm{~nm}$ spacing, quite sufficient for our system. The selected receiving carrier heterodyne-detects the information carried by the corresponding transmitting carrier. Subsequent RF tuning further extracts one of the subcarrier information. It is

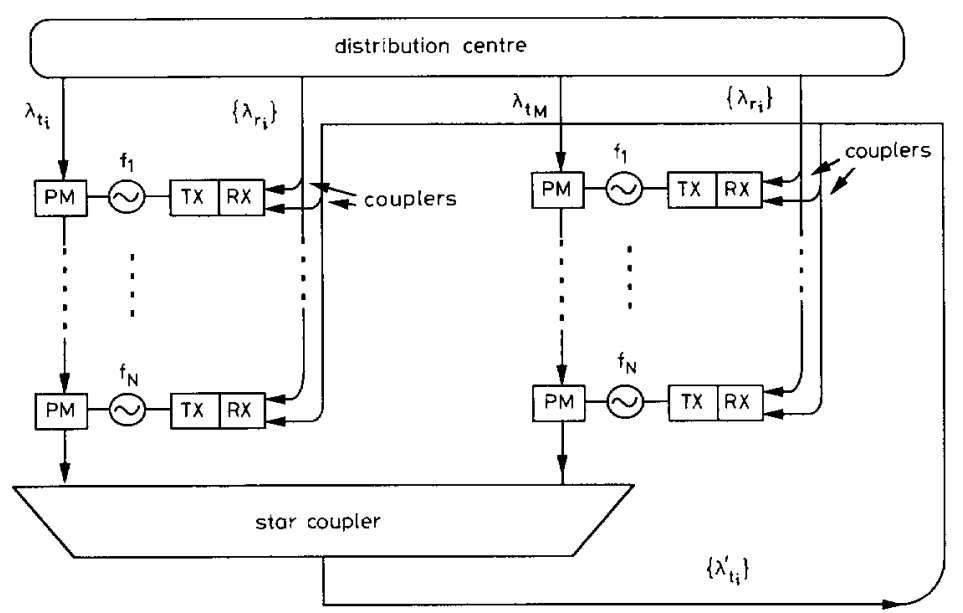

Fig. 7 Schematic diagram of a multiwavelength transparent network

$\lambda_{t 1}, \ldots, \lambda_{i_{m}}=$ unmodulated transmitting carrier set

$\left\{\lambda_{z}^{\prime}\right\}=$ modulated transmitting carriers

$\left\{\lambda_{\mathrm{r}}\right\}=$ receiving carrier set

IEE Proc-Optoelectron., Vol. 141, No. 1, February 1994 
clear that with the present structure a transparent network with $M \times N=800$ nodes can be constructed.

In addition to provide interconnection between network nodes, video distribution can be accomplished in the WRN. An example using the registered wavelengths for video distribution is illustrated in Fig. 8. The transmitting carriers $\left\{\lambda_{t i}\right\}$ coming from the distribution centre are separated by a bank of optical filters; each carrier is then phase-modulated by $N$ subcarriers carrying video programmes $\left(\mathrm{CH}_{1} \sim \mathrm{CH}_{N}\right)$. The carriers are combined and are distributed to many subscribers. The receiver at the subscriber's premises again uses a tunable optical filter (not shown) to select one of the receiving carriers to detect the information carried by a specific transmitting wavelength, and RF tuning is employed to select one of the programmes. With this configuration, each subscriber can actually choose one of the $M \times N$ programmes from the video centre.

The above are merely several examples of communication networks; many other structures are implementable in the WRN. The main difference between the WRN and most of the proposed networks is that the carriers in the WRN are supplied by the WGC but not locally generated at network nodes. This releases network nodes from generating stable and narrow linewidth carriers; just fixed or tunable filters are used to select the desired carriers. The carrier wavelengths are very stable and are well identified without ambiguity. Thus, the resultant communication network is expected to be very stable.

\subsection{Photonic switching fabrics}

Photonic switching is a method which promises to break the bottleneck imposed by limited electronic switching speed. Several photonic switching fabrics based either on space, time, wavelength, or a combination of these are under intensive study [12]. Here the possible photonic switching fabrics in the WRN is discussed.

Fig. 9 shows a photonic switching system based on the two carrier sets. The incoming transmitting carrier set is split into $M$ paths and received by $M$ receivers. Each

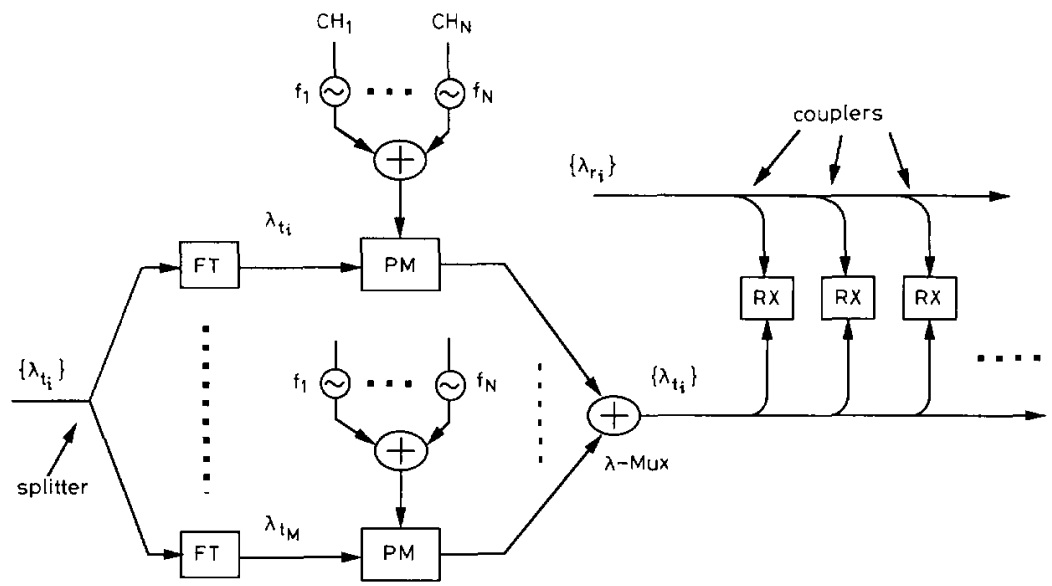

Fig. 8 Video distribution system in the WRN

FT $=$ optical filter

Each filter selects one transmitting carrier which is then modulated by $\mathrm{N}$ subcarriers carrying $\mathrm{N}$ programmes $\left(\mathrm{CH}_{1}-\mathrm{CH}_{\mathrm{s}}\right)$. A receiver selects the desired transmitting carrier by an optical filter, and RF tuning is performed to select one of the programmes

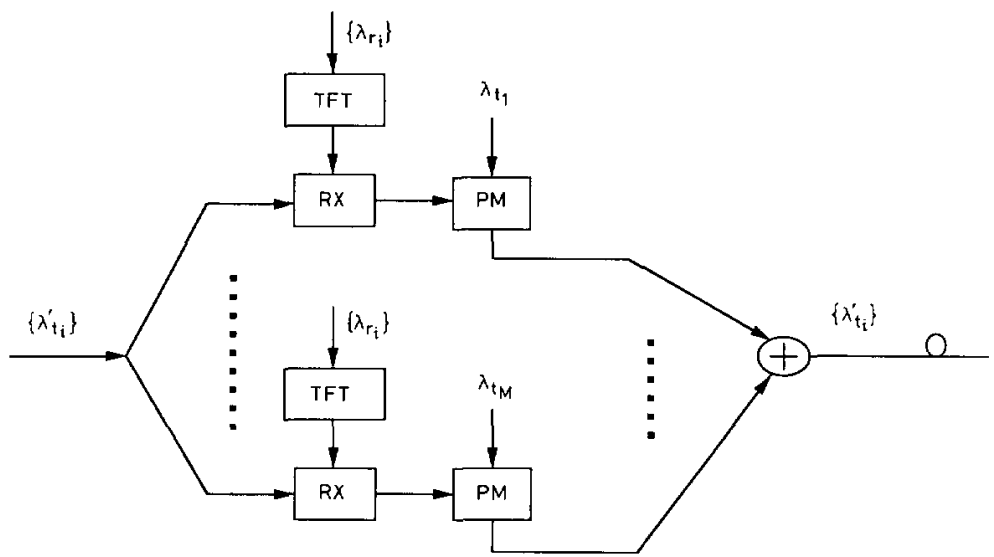

Fig. 9 Photonic switching system featuring tunable receiving carrier

TFT $=$ tunabie optical filter

The receiver selects one of the transmitting carriers by tuning the TFT to the corresponding receiving carrier. Then the detected information is put on to a new transmitting carrier to complete the switching function 
receiver uses a tunable optical filter (TFT) to extract a desired receiving carrier from $\left\{\lambda_{r}\right\}$ to detect the information carried by a specific transmitting carrier. The detected signal then phase-modulates one of the transmitting carriers to transfer the information to the new carrier. In this manner, we have a wavelength interchanger between $M$ carriers which is strictly nonblocking. The switching system also features multicasting capability because the information carried by a specific input wavelength can be distributed to more than one output wavelength. An alternative switching fabric featuring fixed receiving wavelength and tunable transmitting wavelength is shown in Fig. 10. Here the information of a specific transmission wavelength is detected by the corresponding receiving wavelength and is then transfered to the other transmitting wavelength by a TFT and a phase modulator. However, because wavelength conflict occurs when two tunable optical filters select the same transmitting wavelength, the $M$ tunable optical filters must select different transmitting wavelengths. Thus, a multicasting function is not present. The former method is preferable because it does provide a multicasting capability.

The photonic switching fabric can be extended to accommodate high-capacity switching. Fig. 11 shows a three-stage switching fabric which provides wavelength interchange between $M$ input fibres each having $M$ registered wavelengths. In Fig. 11 the $\lambda$-switch is the same as that shown in Fig. 9. The multistage switching system has the same structure as that shown in Reference 27 but here the $\lambda$-switch is based on optical phase modulation. There are three stages of $\lambda$-switches. An inter-stage network consisting of a filter bank and a star coupler is employed to connect two stages. The filter bank is composed of $M$ fixed-wavelength filters to separate the $M$ wavelengths and the star coupler is used to combine the $M$ wavelengths coming from different filter banks. With the interstage network, each $\lambda$-switch has potential connectivity to every next stage $\lambda$-switch. In such a photonic switching system, wavelength stability is of paramount importance to avoid wavelength conflict and interference. Since all wavelengths in the WRN are stable and are well identified, the resultant switching system is expected to be reliable even though a large number of wavelengths are involved. With the multistage switching shown in Fig. 11 we can provide potential connectivity between $M^{2}$ wavelengths.

\section{Advantages}

There are many advantages associated with the proposed WRN

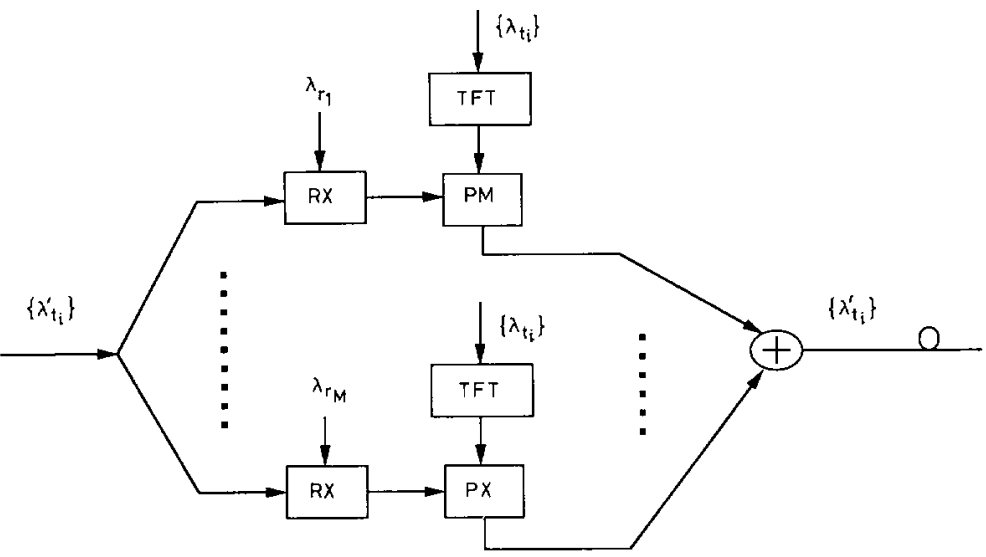

Fig. 10 Photonic switching system featuring tunable transmitting carrier

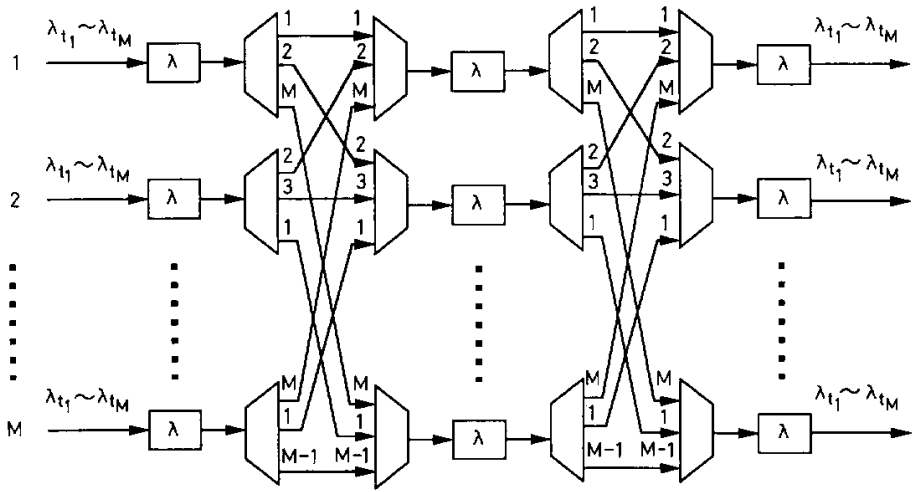

Fig. 11 Three-stage high-capacity photonic switching system

Each $\lambda$-switch corresponding to a single-stage switch as Fig. 9

$\square=$ filter bank

D = star coupler

IEE Proc-Optoelectron., Vol. 141, No. 1, February 1994 
(a) Standardisation of optical and electrical domains: In conjunction with the timing synchronisation provided by the Synchronous Optical NETwork (SONET) standard, the WRN will be a multiwavelength optical network standardised both in wavelength and timing, i.e. both the optical and electrical domains are standardised. The most important implication of the WRN is that we can standardise future multiwavelength networks through wavelength registration and timing synchronisation. Once wavelength and timing are both standardised, all the optical and electrical interfaces are unified, which is the key step toward a standard multiwavelength network

(b) Standardisation of optical components: All optical components used in the multiwavelength network, such as fixed/tunable filters, wavelength Mux/Demux, modulators, optical amplifiers, etc., can be standardised via wavelength registration, in turn mass production resulting in significant cost reduction.

(c) Free from wavelength ambiguity: In the WRN, high-speed WDM-based photonic switching can be implemented without a wavelength identification problem. Also, wavelengths can be freely used as addressing and routing elements without ambiguity.

(d) Ease of carrier generation and control: With the centralised-light-source architecture, we have to deal only with two carrier sets in the WGC; a stable multiwavelength optical network is then constructed without circumstantial wavelength control at every network node. Alternatively, it would be a cumbersome job with distributed-light-source architecture and it is very hard to achieve wavelength registration.

With these advantages, we believe that the WRN is a feasible structure for future multiwavelength optical networks.

\section{Conclusions}

A novel multiwavelength network, the WRN, that is a wavelength-registered network was investigated. Unlike most of the proposed multiwavelength networks that adopt distributed-light-source architecture that inevitably involves a large number of semiconductor lasers, the WRN uses only a stable reference laser to generate all the carriers needed for the entire network. The WRN is wavelength-registered so all carrier wavelengths are well identified without ambiguity. It is also a stable network with very little wavelength drift.

The central idea of WRN is to use two stable carrier sets as the 'carrier supplies' for all the network nodes. It is a centralised-light-source architecture differing significantly from the widely-adopted distributed-light-source architecture. The centralised-light-source architecture makes a wide-area wavelength-registered network possible. Indeed, the WRN has many advantages and is a possible contributor toward a standard wide-area multiwavelength network.

To implement the WRN, several issues should be considered. A conceptual study of the components included: (a) the WGC, the centre generating the carriers; (b) the feeder network, the network distributing the carriers from the WGC to network nodes; (c) the communication network, the network actually performing information exchange; and (d) the photonic switching fabric, the switching system executing fast optical signal switching. Optical phase modulation is widely adopted in the WRN. A phase-modulation based multicarrier generator for carrier generation was used and a phase-modulated SCM adopted for information delivery. The use of SCM provides a flexible transmission and enables the sharing of optical carriers by many nodes. Also, phase modulation preserves a constant envelope that eases optical amplification. A 40-wavelength network was considered with each wavelength carrying 20 subcarriers; a total of $160 \mathrm{Gbps}$ information is estimated for a single-mode fibre.

The salient feature of WRN is the wavelength registration. Network standardisation is a trend, SONET is an example. SONET intends to synchronise timing in the global optical network such that interconnection between different networks can be standardised. However, to construct a standard multiwavelength optical network in the future, not only timing synchronisation but also wavelength should be standardised. In this aspect, the WRN is a very good solution for a wavelength-standardised network. Once wavelength and timing are both standardised, both the optical and electrical domains will be standardised. This will result in a standard multiwavelength network with interconnections within the network that are well specified both in the optical and electrical domains. Thus the WRN is a feasible architecture toward a standard multiwavelength network in the future.

\section{References}

1 BRACKETT, C.A.: 'Dense wavelength division multiplexing networks: Principles and applications', IEEE J. Sel. Areas Commun. 1990,8 , pp. 948-966

2 GOODMAN, M.S.: 'Multiwavelength networks and new approaches to packet switching', IEEE Commun. Mag., 1990, 27, pp. 27-35

3 KOBRINSKI, H., BULLEY, R.M GOODMAN, M.S, VECCHI, M.P BRACKET, C.A. CURTIS, L., and GIMLETT, J.L.: "Demonstration of high capacity in the LAMBDANET architecture: 'Demonstration of high capacity in the LAMBDANET architecture: A multiwav

284-286

4 ARTHURS, E., GOODMAN, M.S., KOBRINSKI, H., and VECCHI, M.P.: 'HYPASS: An optoelectronic hybrid packetswitching system', IEEE J. Sel. Areas Commun., 1988, 6, pp. 15001510

5 GLANCE, B., POLLOCK, K., BURRUS, C.A., KASPER, B.L. EISENSTEIN, G., and STULZ, L.: 'Densely spaced WDM coherent optical star networks', Electron. Lett, 1987, 23, pp. 875-876

6 BACHUS, E.J., BRAUN, R.P., CASPAR, C., FOISEL, H.M., GROBMANN, E, STREBEL, B., and WESTPHAL, F.J.: 'Coherent optical multicarrier systems', J. Lightwave Technol, 1989, 7, pp. 375-384

7 LIEW, S.C., and CHEUNG, K.W.: 'A broad-band optical network based on hierarchical multiplexing of wavelengths and RF subcarriers', J. Lightwave Technol., 1989, 7, pp. 1825-1838

8 IRSHID, M.I., and KAVEHRAD, M.: 'A fully transparent fiberoptic ring architecture for WDM networks', J. Lightwave Technol. 1992, 10, pp. 101-108

9 GROSS, R., OLSHANSKY, R., and HILL, P.: '20 channel coherent FSK system using subcarrier multiplexing', IEEE Photonics Technol. Lett., 1989, 1 , pp. 224-226

10 OLSHANSKY, R., GROSS, R., and SCHMIDT, M.: 'Subcarrier multiplexed coherent lightwave systems for video distribution', IEEE J. Sel. Areas Commun., 1990, 8, pp. 1268-1275

11 CIMINI, L.J.: 'Optical phase division multiplexing for local communications applications', IEEE Trans. Commun., 1989, 37, pp. $1282-1292$

12 HINTON, H.S.: 'Photonic switching fabrics', IEEE Commun. Mag., 1990, 28, pp. 71-89

13 MINISCALCO, W.J.: 'Erbium-doped glasses for fiber amplifiers at $1500 \mathrm{~nm}$ ', J. Lightwave Technol, 1991, 9, pp. 234-250

14 RYU, S., YAMAMOTO, S., TAGA, H., EDAGAWA, N., YOSHIDA, Y., and WAKABAYASHI, H.: 'Long-haul coherent optical fiber communication systems using optical amplifiers', $J$. Lightwave Technol., 1991, 9, pp. 251-260

15 INOUE, K., TOBA, $H$., and NOSU, K.: 'Multichannel amplification utilizing an $\mathrm{Er}^{+3}$-doped fiber amplifier', $J$. Lightwave Technol, 1991,9 , pp. $368-374$ 
16 KAZOVSKY, L.G.: 'Phase- and polarization-diversity coherent optical techniques', J. Lightwave Technol., 1989, 7, pp. 279-292

17 RYU, S., and HORIUCHI, Y.: 'Use of an optical amplifier in coherent receiver', IEEE Photonics Technol. Lett., 1991, 3, pp. 663665

18 GROSS, R., and OLSHANSKY, R.: 'Multichannel coherent FSK experiments using subcarrier multiplexing techniques', $J$. Lightwave Technol., 1990, 8, pp. 406-415

19 MAEDA, M.W., BARRY, J R KUMAZAWA, T, and WAGNER, R.E.: 'Frequency identification and stabilization of packaged DFB lasers in $1.5 \mu$ m region'. OFC ' 89 , Houston, TX, USA, 1989

20 KAZOVSKY, L.G.: 'Optical signal processing for lightwave communications networks', IEEE J. Sel. Areas Commun., 1990, 8, pp 973-982

21 WALKER, G.R., WALKER, N.G., STEELE, R.C., CREANER M.J., and BRAIN, MC. 'Erbium-doped fiber amplifier cascade for multichannel coherent optical transmission', J. Lightwave Technol. 1991,9 , pp. 182-192
22 STOLEN, R.H.: 'Nonlinearity in optical fibers', Proc. IEEE, 1980, 68, pp. 1232-1236

23 CHRAPLYVY, A.R.: 'Limitations on lightwave communications imposed by optical-fiber nonlinearities', J. Lightwave Technol., 1990, 8, pp. $1548-1557$

24 CHRAPLYVY, A.R., and HENRY, P.S.: 'Performance degradation due to stimulated Raman scattering in wavelength-division-multiplexed optical-fiber systems', Electron. Lett., 1983, 19, pp. 641-643

25 CHRAPLYVY, A.R., MARCUSE, D., and HENRY, P.S.: 'Carriedinduced phase noise in an angle-modulated optical-fiber systems', $J$. induced phase noise in an angle-modula

26 HIRABAYASHI, K., TSUDA, H., and KUROKAWA, T.: 'Tunable wavelength-selective liquid crystal filters for 600-channel FDM systems', IEEE Photonics Technol. Lett., 1992, 4, pp. 597-599

27 SUZUKI, S., NISHIO, M., NUMAI, T., FUJIWARA, M., ITOH, M., MURATA, S., and SHIMOSAKA, N.: 'A photonic wavelengthdivision switching system using tunable laser diode filters', J. Lightwave Technol., 1990, 8, pp. 660-665 